\title{
Accessory Nerve Schwannoma Extending to the Foramen Magnum and Mimicking Glossopharyngeal Nerve Tumor-A Case and Review of Surgical Techniques
}

\author{
Seidu A. Richard ${ }^{1,2}$, Zhi Gang Lan', Yuekang Zhang ${ }^{1 *}$, Chao You ${ }^{1 *}$ \\ ${ }^{1}$ Department of Neurosurgery, Post-Graduate Training Centre, West China Hospital, Sichuan University, Chengdu, China \\ ${ }^{2}$ Department of Immunology, Jiangsu University, Zhenjiang, China \\ Email: ^2012zykyx@sina.cn, ^youchao@vip.126.com
}

How to cite this paper: Richard, S.A., Lan, Z.G., Zhang, Y.K. and You, C. (2017) Accessory Nerve Schwannoma Extending to the Foramen Magnum and Mimicking Glossopharyngeal Nerve Tumor-A Case and Review of Surgical Techniques. World Journal of Neuroscience, 7, 233-243. https://doi.org/10.4236/wjns.2017.73019

Received: May 19, 2017

Accepted: June 17, 2017

Published: June 20, 2017

Copyright $\odot 2017$ by authors and Scientific Research Publishing Inc. This work is licensed under the Creative Commons Attribution International License (CC BY 4.0).

http://creativecommons.org/licenses/by/4.0/

\section{(c) (i) Open Access}

\begin{abstract}
Background: Intracranial schwannomas of the accessory nerve are very rare lesions. They are categorised according to their locations into either intrajugular or intracistemal schwannomas although most of them are intrajugular. The intrajugular type constitutes about $2 \%$ to $4 \%$ of all intracranial schwannomas described in literature. Aim: It's very unusual for an accessory nerve to mimic glossopharyngeal nerve looking at the anatomical location of the accessory nerve. Although many authors have written on accessory nerve, none have described this unusual presentation. We present a case, management as well as review on the classification and appropriate surgical techniques we could have use to access the tumor in our patient since the choice of a particular surgical approach is based on the nature of tumor, location as well as it extension into other adjacent structures. Case Presentation: We present a case of 52-year-old woman with very unusual accessory nerve schwannoma which mimics the clinical presentation of glossopharyngeal nerve tumor. The main symptom in our case is six (6) months history of deviation of the tongue to right side with dizziness and change of voice. Conclusion: The unusual presentation in our case could be due to massive compression of glossopharyngeal nerve by the growing accessory nerve schwannoma since most lower cranial nerve schwannomas at this location will almost always course compressive symptoms.
\end{abstract}

\section{Keywords}

Schwannoma, Accessory Nerve, Glossopharyngeal Nerve, Suprajugular, Retrosigmoid, Translabyrinthine 


\section{Introduction}

Intracranial schwannomas although very rare, are made up of roughly $8 \%$ of all primary brain tumors [1] [2] of which accessory nerve (AN) schwannomas form a part [3] [4]. The origins of these tumours are usually very difficult to predict during preoperatively assessment of patients [5]. Careful intra-operative observation has led to the classification of AN schwannomas into extracranially or intracranially base on the roots of the never [4]. Many authors have further categorized the intracranial types above into intrajugular schwannomas since they usually extend into the jugular foramen and make up the majority of cases, and intracisternal schwannomas because there are located at the cisterna magna [3] [6] [7]. The most common clinical presentations of most schwannomas extending to the jugular region are cranial nerve dysfunction, such as hearing impairment, dysphagia, and difficulty in phonation although the clinical manifestations of these tumors vary in relations to tumor location and the magnitude of their growth [8], while intracisternal tumors present with cerebellar signs and myelopathy [3] [9]. We present a case and also review the appropriate surgical techniques we could have use to access the tumor in our patient since the goal of surgical approach is to have enough expose to the lesion, no matter how extended the tumor maybe. The unusual presentation in our case could be massive compression of glossopharyngeal nerve by the growing AN schwannoma since most tumors at this location will almost always course compressive symptoms.

\section{Case Report}

A 52-year-old female presented with six [6] months history of acute deviation of the tongue to right side with dizziness and change of voice. The symptoms started 10 years ago with headache which she presented at outpatient's department (OPD) and a computer topographic scan (CT scan) examination revealed a small lesion at the posterior fossa. The lesion has been increasing in size for over this 10 years' period with any no symptoms until this acute presentation above. Neurological examination on admission revealed no focal neurological deficit, except for tongue deviating to the right side of her body. She had no significant medical or surgical history. Initial MRI scan done at a local hospital 10 years ago revealed a small mass at the right posterior fossa which she refused surgery. MRI scan done at our facility demonstrated a 4.2 $\mathrm{cm} \times 2.9 \mathrm{~cm} \times 3.6 \mathrm{~cm}$ well-circumscribed mass with high and nonhomogeneous signal intensity close to the right foramen of luschka and extending down to the foremen magnum. It compressed the adjacent medulla and distorted the fourth ventricle (Figure 1). The lateral ventricles are not dilated and no hydrocephalus.

After general anesthesia, the patient was put in the park-bench position. Routing use of inferior cranial nerves monitoring was used to maximize the preservation of those nerves. A hockey-stick shaped paramedian incision was made. 
A high-speed miller was used to perform a suboccipital craniotomy. Additionally, posterior rim of foramen magnum and the lamina of $\mathrm{C} 1$ were also
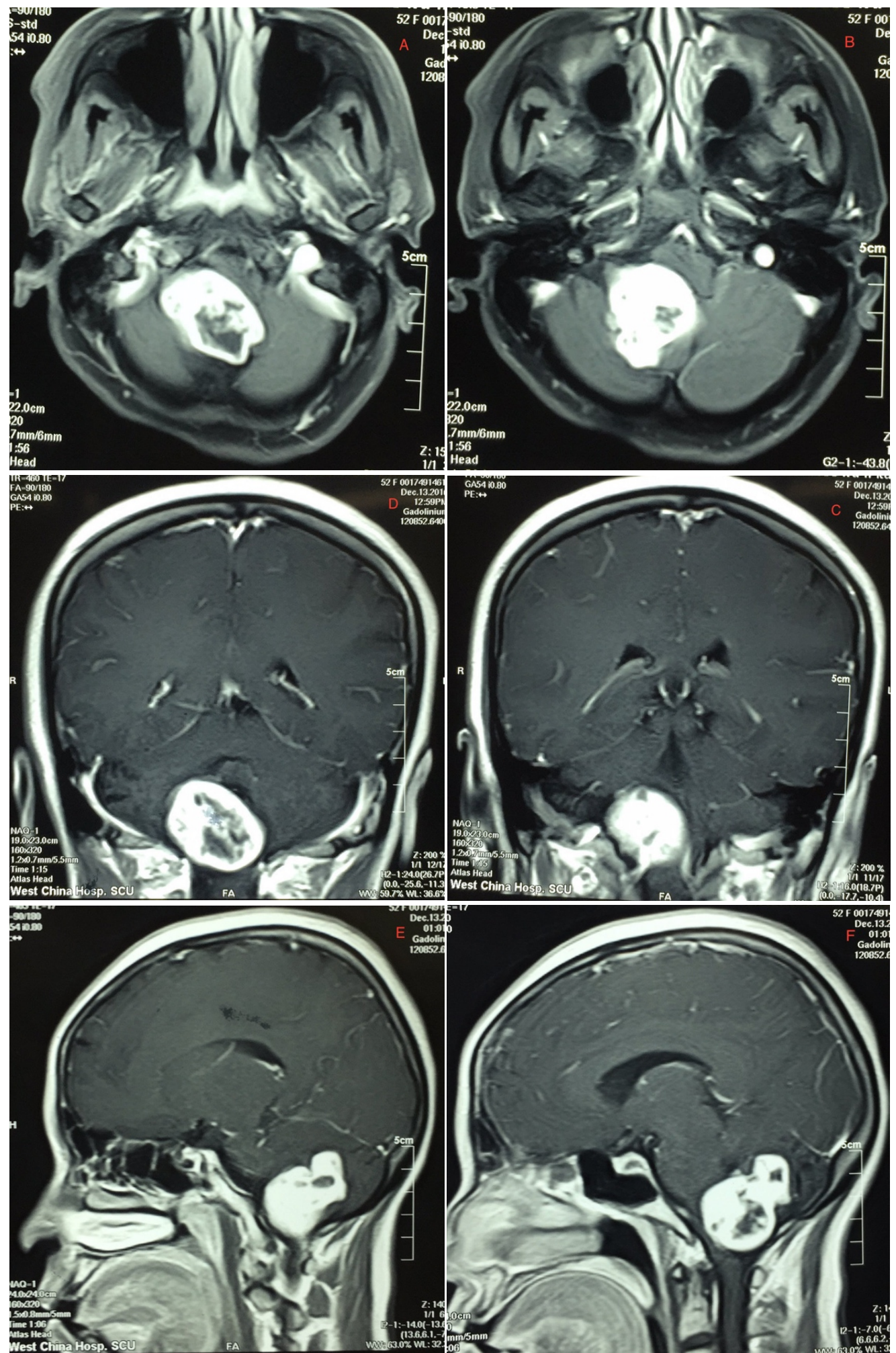

Figure 1. Are axial (A \& B), coronal (C \& D) and sagittal (E \& F) enhanced T1-MRI images showing the tumor in the posterior fossa and extending down to the foramen magnum. The tumor also compresses and displaced surrounding structures. 

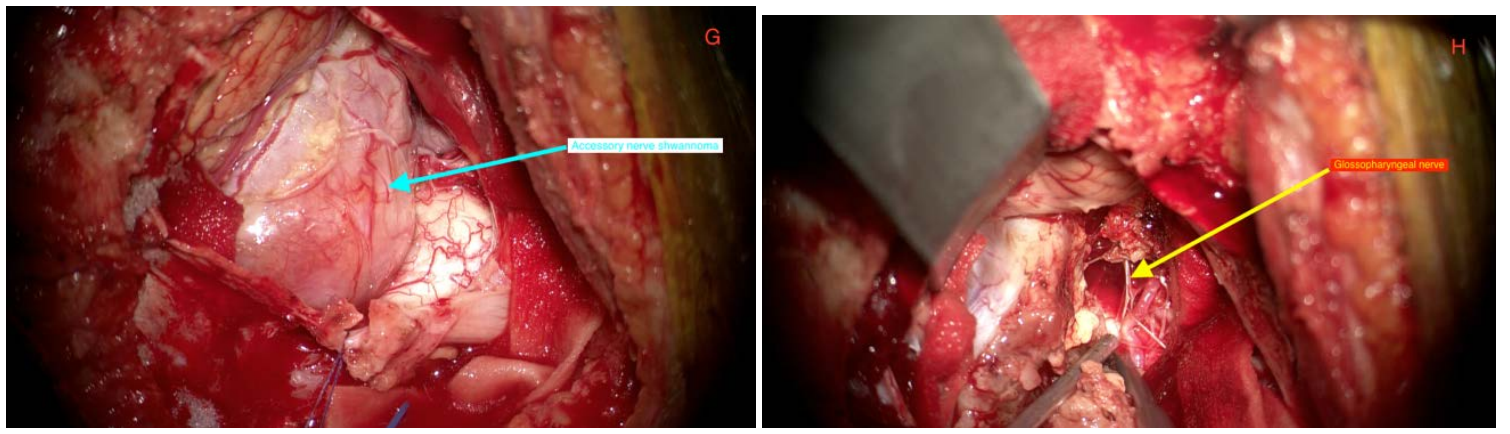

Figure 2. Are intra-operative images showing the tumor after opening the dura $(\mathrm{G})$ and the glossopharyngeal nerve after tumor resection.

removed to gain of wider exposure of the tumor. After placing several tack-up stitches to the dura, the tumor was observed lying between jugular foramen and the foramen magnum Figure 2. Intra-operatively, we noticed that the lesion was tightly adhered to the glossopharyngeal nerve (GPN) but the origin was the AN and needed a much more experienced surgeon. After careful debulking and separation of the tumour by the head of our team, the lesion was completely resected without any cranial nerves dysfunction Figure 2. Pathological examination of the surgical specimen confirmed the diagnosis of benign schwannoma. Histologically, we observe solid areas of parallel spindle cells with eosinophilic cytoplasm and $\mathrm{S} 100(+)$, Nestin(+) which confirms the diagnosis of schwannoma. Post-operative imaging show total tumour resection. The patient had a good recovery and discharge home on the seventh $\left(7^{\text {th }}\right)$ day after operation. The tongue deviation improved markedly. A scheduled review was arranged on the sixth month after operation.

\section{Discussion}

Accessory nerve (AN) schwannomas are extremely uncommon and it is genuinely puzzling to recognize the origin of tumours preoperatively [5]. To the best of our knowledge no author has presented a case AN schwannoma with a history of deviation of the tongue to right side with dizziness and change of voice. Some authors have indicate the difficulties of identifying the origin of tumors arising from the $9^{\text {th }}, 10^{\text {th }}$ and $11^{\text {th }}$ cranial nerves even at operation [5]. We were able to identify the origin of the tumour because it was tightly adhere to glossopharyngeal never hence needed a more carefully dissection and separation of structure. Also, the tumor was a pedunculated one hence we were able to see the continuity of proximal end towards the brain stem. Julow was the first to classified AN scwannomas into intrajugular schwannomas base on their extension to the jugular foramen and intracisternal schwannomas base on their extension to the cisterna magna [3] [6]. A very small number of authors have described cases of ANs with cranial root origin although several authors have described cases of AN but in most of the cases, the origin of these tumors was the spinal root of the accessory nerve [3] [10]. Intrajugular schwannomas can present with several permutations of clinical symptoms in- 
cluding $5^{\text {th }}$ to $12^{\text {th }}$ cranial nerve palsies, cerebellar signs and/or myelopathy linked to their direct compressive effects on the cerebellum, brain stem, and/or spinal cord. Furthermore, intrajugular tumors typically present with otologic symptoms and jugular foramen syndrome, characterized by symptoms of tinnitus, hearing impairment, dysphagia, and hoarseness [3] [8]. Intracisternal schwannomas generally present with symptoms of accessory nerve palsy, cerebellar signs and myelopathy [3]. It is usually imperative to monitor very vital structures during operation. These intraoperative monitoring consisted of lower CNs, brainstem auditory evoked potentials, somatosensory evoked potentials, motor evoked potentials, and facial nerve monitoring [11].

The location of tumor, the size and presenting symptoms particularly preoperative hearing are special issues the surgeon must consider before choosing a surgical approach [8]. The ultimate surgical approach is one that gives the surgeon a wide access to the whole tumor with minimal brain manipulation and minimal morbidity related to additional $\mathrm{CN}$ deficits as well as single stage total tumor removal [8]. Furthermore, to accomplish a total tumor resection, the surgical procedures should be well designed, since recurrent operation increases the risk of lower cranial nerve injuries and incomplete removal involves inevitable reappearance [8]. The common surgical approaches approach includes post-auricular $\mathrm{C}$-shaped incision, retro and infra-labyrinthine mastoidectomy, total exposure of the jugular bulb, skeletonization of the facial nerve (anterior translocation is rarely if ever performed unless there is a giant tumor), lateral suboccipital craniotomy and trans-condylar/trans-tubercular exposure, exposure of the IJV, trans-jugular and trans-sigmoid approaches; and intra-dural exposure [11] [12]. Sung-won et al. revealed that the histopathological examination of a similar schwannoma case indicated that the tumor was composed of hypercellular (Antoni Type A pattern) and hypocellular (Antoni Type B pattern) areas. The tumor cells were immunopositive for S-100 and immunonegative for glial fbrillary acidic protein [3].

\subsection{Anatomical Study}

The jugular foramen is found amid the lateral part of the occipital bone and the petrous part of the temporal bone [1]. The foramen is described as a canal running anteriorly, inferiorly, and laterally from an intracranial to an extracranial opening [1] [13] and it is divided into two or rarely three compartments [1] [14]. The two compartments are divided by a bony fibrous septum although rarely the separation is incomplete, and usually refered to as "intrajugular process", which may be accompanied by a fascial ligament [1] [14]. The anteromedial compartment is insinuated to as the "pars nervosa," and the posterolateral compartment is termed the "pars venosa" or "pars vascularis". The pars nervosa comprises of the inferior petrosal sinus, vena canaliculi cochleae, and glossopharyngeal nerve, while the pars venosa comprises of the vagus and accessory nerves and the proximal part of the jugular bulb. A current study has indicated that the jugular bulb is not truly grouped into the pars nervosa and pars venosa [1] [15]. Further 
with cadaver dissections indicated that the vascular system was confined within the endothelial-lined lumen and that the cranial nerves were enclosed by fibrous tissue and lay outside the lumen of the jugular bulb [1] [16].

Although several authors divide the jugular foramen into anterior, posterior, and intermediate positions, where the intermediate position contains the ninth, $10^{\text {th }}$, and $11^{\text {th }}$ cranial nerves, there is still no unanimous agreement regarding the position of cranial nerves in the jugular foramen. While some authors are of the view that the $9^{\text {th }}, 10^{\text {th }}$, and $11^{\text {th }}$ cranial nerves are located in the pars nervosa [1] [17] others are of the view that only the $10^{\text {th }}$ and $11^{\text {th }}$ nerves are located in the pars nervosa [1] [14] although it universal accepted that there is a common dural sheath for the $10^{\text {th }}$ and $11^{\text {th }}$ cranial nerves and the glossopharyngeal nerve has a separate dural covering as it passes through the foramen. The meningeal branches of the occipital and ascending pharyngeal arteries pass through the intermediate portion and they are usually the main vascular supply to tumors in this region [1]. Therefore, Jugular foramen schwannomas can arise from the proximal or distal part of the $9^{\text {th }}, 10^{\text {th }}$, and $11^{\text {th }}$ cranial nerves, presenting as either intracranial or extracranial masses [1].

\subsection{Clinical Staging and Classification}

Two types of classification have been established for jugular foramen schwannomas with modifications depending on the site and extent of the tumors. The classification scheme aids in deciding on the operative approach. Franklin and colleagues classified these tumors into Classes A, B, and C, centred on the classifications used for glomus jugular tumors [1] [18]. Kaye and associates established a similar but easier classification system, as follows: Type A tumors were primarily intracranial with minimal extension into the bone; Type $\mathrm{B}$ tumors were primarily within the bone with or without an intracranial component; and Type $C$ tumors were primarily extracranial with only a minor extension into the bone or into the posterior fossa [1] [19]. Pellet and colleagues modified the classification scheme by adding another type, Type $D$, which is a saddlebag-shaped Type B tumor with intracranial and extracranial components linked via the jugular foramen [1] [20].

The most Current classification of jugular foramen schwannomas is according to the radiological and surgical features, the tumors are classified into four types: Type A, a tumor primarily at the cerebellopontine angle with minimal enlargement of the jugular foramen; Type $\mathrm{B}$, a tumor primarily at the jugular foramen with intracranial extension; Type $\mathrm{C}$, a primarily extracranial tumor with extension into the jugular foramen ; and Type $\mathrm{D}$, a dumbbell-shaped tumor with both intra- and extracranial components [1] (Table 1).

Furthermore, Bulsara and associates proposed another scheme which is a modification of the classification system initially proposed by Kaye and colleagues [11] [19] and modified by Pellet and associates [11] [20]. In this scheme (Table 2), the tumors are classified according to only imaging studies to deter- 
Table 1. The most current classification of jugular foramen schwannomas is according to the radiological and surgical features.

\begin{tabular}{cc}
\hline Class & Definition \\
\hline Type A & primarily intracranial: minimal extension into jugular foramen \\
Type B & primarily intraosseous: with or without intracranial extension \\
Type C & primarily extracranial: minimal extension into jugular foramen \\
Type D & saddlebag- or dumbbell-shaped intra- \& extracranial extensions \\
\hline
\end{tabular}

Table 2. Modified classification system for jugular foramen schwannomas as propose by Bulsara and associates using only imaging studies to determine the surgical approach.

\begin{tabular}{ccc}
\hline Grading system & Location of Tumor & Operative approach. \\
\hline Type A & purely Intradural & Retromastoid Approach or ELITE \\
Type B & Intradural and involve the jugular bulb & Intradural and Transjugular approach \\
Type C & $\begin{array}{c}\text { Intradural component and involve } \\
\text { both the jugular bulb and the IJV }\end{array}$ & $\begin{array}{c}\text { Intradural and Transjugular approach } \\
\text { with possible ligation of the IJV. }\end{array}$ \\
\hline
\end{tabular}

mine the surgical approach as follows: Type A, intradural tumor; Type B, dumbbell-shaped tumor; and Type C, dumbbell-shaped tumor with high cervical extension. Bulsara et al. therefore proposed that Type A tumors are purely intradural and can be approached via a retromastoid approach or ELITE. Type B tumors are intradural and involve the jugular bulb; these tumors are best approached via an intradural and transjugular approach. Type $\mathrm{C}$ tumors have an intradural component and involve both the jugular bulb and the IJV. In these tumors, an intradural and transjugular approach with possible ligation of the IJV may be necessary. They indicated that, their modification facilitates operative planning for current skull base surgery techniques and provides a simplified method for reporting and analyzing surgical results [11].

\subsubsection{Suprajugular Approach}

Paulo et al. are credited for the detailed description of this approach. The suprajugular approach is basically a presigmoid infralabyrinthine route. This approach is best used to assess tumors extending anteriorly to the jugular bulb. A post-auricular incision is made, and the internal carotid artery (ICA), external carotid artery (ECA), internal jugular vein, and the ninth, $10^{\text {th }}, 11^{\text {th }}$, and $12^{\text {th }}$ cranial nerves in the cervical region are identified. The sternocleidomastoid muscle is dissected, mobilized, and reflected inferiorly. A mastoidectomy is followed by complete skeletonization of the sigmoid sinus, jugular bulb, and jugular vein. The presigmoid, infralabyrinthine space is opened and the dura mater is identified superior to the patent jugular bulb and inferior to the labyrinth. After the cerebellomedullary cistern is opened, releasing cerebrospinal fluid, the tumor is exposed. The lesion is debulked with suction and bipolar coagulation or by using the ultrasonic aspirator. The lower cranial nerves $\left(9^{\text {th }}-12^{\text {th }}\right)$, the posteroinferior cerebellar artery, the anteroinferior cerebellar artery, and the vertebral artery are dissected away from the tumor through the arachnoid plane, and 
the lesion is radically removed [21]. This approach is the most appropriate for our case considering the location of the lesion. We however skeletonized $\mathrm{C} 1$ to gain more assess to the lesion in the foramen magnum.

\subsubsection{Retrosigmoid Approach}

Chamoun et al. are credited for the detailed description of this approach. They indicated that, an incision is made approximately 2 fingerbreadths behind the pinna of the ear. A suboccipital craniectomy is performed, and the edges of the transverse and sigmoid sinuses are exposed. The air cells are carefully waxed to prevent CSF leakage. The dura mater is then opened in a cruciate fashion such that there is a flap superiorly and laterally to allow flat trajectory along the axis of the sigmoid sinus, and the cerebellum is gently retracted to expose the tumor in the CPA. The posterior lip of the IAC is drilled until the transverse crest can be palpated with a hook. The drilling is performed using a diamond drill bit and with copious irrigation to prevent thermal injury. The retrosigmoid approach exposes $180^{\circ}$ of the circumference of the IAC. During drilling, extensive irrigation is used to avoid any thermal damage to the nerves in the canal. Air cells at this level can be opened during the drilling and should also be waxed to prevent CSF leakage [22].

The vestibular nerves are divided, and the facial nerve is identified anterior to them and confirmed with stimulation. The cochlear nerve is identified just inferolateral to the facial nerve. Specific techniques for preserving the facial and vestibular nerves when dissecting from the tumor include gentle dissection using fine otology instruments. The tumor is dissected from medial to lateral to avoid traction on the cochlear nerve as it exits the perforated bone at the end of the IAC. Careful dissection is performed under direct visualization, and the interface of the nerve with the tumor is dissected under high magnification. Both facial nerve and brainstem auditory evoked response monitoring are used during this dissection. The retrosigmoid approach permits removal of tumors of different sizes and offers the possibility of hearing preservation [22]. Many experienced neurosurgeons prefer this a pproach for tumors with significant mass in the cistern and in patients with serviceable hearing, in whom hearing preservation is the goal. This approach gives the surgeon a wide view of the cisternal component of the tumor and thus good access to the root entry zone of the acoustic nerve. The approach usually requires cerebellar retraction and less access to the facial and cochlear nerves in the distal IAC, which increases the potential to leave a residual tumor fragment behind [22]. However, this approach is not appropriate for our case considering the location of the lesion.

\subsubsection{Translabyrinthine Approach}

Chamoun et al. are credited for the detailed description of this approach. They again indicayed that, the patient is positioned supine, and the head is rotated to the contralateral side. Somatosensory evoked potentials, motor evoked potentials, and facial nerve monitoring are used. A hockey stick-shaped retroauricular skin incision extending behind the mastoid tip is made. The mastoidectomy is 
performed with the high-speed drill, and the sigmoid sinus, presigmoid dura mater, and middle fossa dura are exposed. The mastoid segment of the facial nerve is skeletonized. The eustachian tube is packed with bone wax and Surgical to prevent CSF leakage. Next, the labyrinthectomy is performed to access the IAC. The translabyrinthine approach exposes $270^{\circ}$ of the circumference of the IAC. The dura of the IAC is then opened. We prefer to open along the axis of the IAC and use a Y-shaped incision to open the dura in the posterior fossa. The facial nerve is located and confirmed using stimulation. The vestibular nerves are cut, and the tumor is dissected from the facial nerve. The presigmoid dura is then opened. The tumor is dissected from the cerebellum and the brainstem and is debulked using the ultrasonic aspirator. The translabyrinthine approach is the most preferred choice for patients with poor preoperative hearing and for patients with large tumors who have a low probability of hearing preservation. This approach offers early identification of the facial nerve in the auditory canal with absolutely no need for cerebellar retraction [22]. The size of the tumor is typically not a limiting factor for this approach. However, this approach is also not appropriate for our case considering the location of the lesion.

\section{Conclusion}

Intracranial accessory nerve schwannomas are exceedingly rare. Clinical symptoms generally consist of accessory nerve palsy, cerebellar signs, and myelopathy. Our case is special because of the unusual presentation which will be as a result of massive compression of glossopharyngeal nerve by the growing AN schwannoma. The detection of the exact nerve of origin is usually determined during operation although MRI is very helpful in the initial diagnosis. With appropriate preoperative evaluation and careful planning of the perioperative period, these tumors can be safely removed and the neurovascular structures preserved without creating new neurological deficits. Intraoperative cranial nerve monitoring is usually very crucial during operation to minimise damage to the adjacent cranial nerves.

\section{Acknowledgements}

Not applicable.

\section{Declaration}

Ethics approval and consent to participate: The ethical committee of the hospital full approved our case study. The patient was informed about our intension to involve her in a case study and she agreed to partake in the study. She signed the concern form before the operation was carried out according to all surgical protocols.

Consent for publication: The patient was dually informed about our intention to publish his case and she fully concerted to the use of these documents. The hospital also concerted to the use of this information for publication.

Availability of data and material: The folder or data is the property of the 
hospital and readily available with proper concern but for confidentiality of the patient the folder cannot to release to third party.

\section{Competing Interests}

All the authors have no competing interest to disclose.

\section{Funding}

Not applicable.

\section{Authors' Contributions}

All the authors contributed equally to the manuscript design and writing.

\section{References}

[1] Samii, M., Babu, R.P., Tatagiba, M. and Sepehrnia, A. (1995) Surgical Treatment of Jugular Foramen Schwannomas. Journal of Neurosurgery, 82, 924-932.

https://doi.org/10.3171/jns.1995.82.6.0924

[2] Nowak, A., Dziedzic, T., Czernicki, T., Kunert, P. and Marchel, A. (2014) Surgical Treatment of Jugular Foramen Meningiomas. Neurologia I Neurochirurgia Polska, 48, 188-195. https://doi.org/10.1016/j.pjnns.2014.05.004

[3] Jin, S.W., Park, K.J., Park, D.H. and Kang, S.H. (2014) Intracisternal Cranial Root Accessory Nerve Schwannoma Associated with Recurrent Laryngeal Neuropathy. Journal of Korean Neurosurgical Society, 56, 152-156. https://doi.org/10.3340/jkns.2014.56.2.152

[4] Al-Ajmi, A.M., Rousseff, R.T., Shamov, T., Ismail, M.J. and Sayer, F.T. (2015) Isolated Spinal Accessory Neuropathy and Intracisternal Schwannomas of the Spinal Accessory Nerve. Interdisciplinary Neurosurgery, 2, 51-53.

https://doi.org/10.1016/j.inat.2015.01.001

[5] Lanotte, M., Massaro, F., Scienza, R. and Faccani, G. (1994) Intracisternal Schwannoma of the Spinal Accessory Nerve Presenting as a Normal Pressure Hydrocephalus Syndrome. Case Report and Review of the Literature. Neurosurgical Review, 17, 225-227. https://doi.org/10.1007/BF00418440

[6] Julow, J. (1983) Neurinoma of Spinal Accessory Nerve. Acta Neurochirurgica, 69, 219-224. https://doi.org/10.1007/BF01401808

[7] Tatebayashi, K., Tanaka, Y., Numata, H., Kawakami, S., Kamitani, H. and Watanabe, T. (2003) Schwannoma of the Spinal Accessory Nerve in the Cisterna Magna. Surgical Neurology, 59, 217-222. https://doi.org/10.1016/S0090-3019(02)01035-2

[8] Bakar, B. (2008) The Jugular Foramen Schwannomas: Review of the Large Surgical Series. Journal of Korean Neurosurgical Society, 44, 285-294.

https://doi.org/10.3340/jkns.2008.44.5.285

[9] Leonetti, J.P., Anderson, D.E., Marzo, S.J., Origitanom, T.C. and Shirazi, M. (2006) Intracranial Schwannomas of the Lower Cranial Nerves. Otology \& Neurotology, 27, 1142-1145. https://doi.org/10.1097/01.mao.0000235309.95313.d3

[10] Tsukamoto, H., Hikita, T. and Takaki, T. (1994) Cerebellopon Tine Angle Meningioma Associated with Cranial Accessory Nerve Neurinoma-Case Report. Neurologia Medico-Chirurgica, 34, 225-229. https://doi.org/10.2176/nmc.34.225

[11] Bulsara, K.R., Sameshima, T., Friedman, A.H. and Fukushima, T. (2008) Microsurgical Management of 53 Jugular Foramen Schwannomas: Lessons Learned Incor- 
porated into a Modified Grading System. Journal of Neurosurgery, 109, 794-803. https://doi.org/10.3171/JNS/2008/109/11/0794

[12] Liu, J.K., Sameshima, T., Gottfried, O.N., Couldwell, W.T. and Fukushima, T. (2006) The Combined Transmastoid Retro-And Infralabyrinthine Transjugular Transcondylar Transtubercular High Cervical Approach for Resection of Glomus Jugulare Tumors. Neurosurgery, 59, 115-125. https://doi.org/10.1227/01.neu.0000220025.81500.8d

[13] Di Chiro, G., Fisher, R.L. and Nelson, K.B. (1964) The Jugular Foramen. Journal of Neurosurgery, 21, 447-460. https://doi.org/10.3171/jns.1964.21.6.0447

[14] Bossy, J. (1970) Atlas of Neuroanatomy and Special Sense Organs. American Journal of Ophthalmology, 70, 862-863. https://doi.org/10.1016/0002-9394(70)90524-6

[15] Kveton, J.F. and Cooper, M.H. (1988) Microsurgical Anatomy of the Jugular Foramen Region. Otology \& Neurotology, 9, 109-112. https://doi.org/10.1097/00129492-198803000-00003

[16] Schwaber, M.K., Netterville, J.L. and Maciunas, R. (1990) Microsurgical Anatomy of the Lower Skullbase-A Morphometric Analysis. American Journal of Otology, 11, 401.

[17] Arenberg, I.K. and McCreary, H.S. (1971) Neurilemmoma of the Jugular Foramen. The Laryngoscope, 81, 544-557. https://doi.org/10.1288/00005537-197104000-00005

[18] Franklin, D.J., Moore, G.F. and Fisch, U. (1989) Jugular Foramen Peripheral Nerve Sheath Tumors. The Laryngoscope, 99, 1081-1087. https://doi.org/10.1288/00005537-198910000-00017

[19] Kaye, A.H., Hahn, J.F., Kinney, S.E., Jr, H.R. and Bay, J.W. (1984) Jugular Foramen Schwannomas. Journal of Neurosurgery, 60, 1045-1053. https://doi.org/10.3171/jns.1984.60.5.1045

[20] Pellet, W., Cannoni, M. and Pech, A. (1988) The Widened Transcochlear Approach to Jugular Foramen Tumors. Journal of Neurosurgery, 69, 887-894. https://doi.org/10.3171/jns.1988.69.6.0887

[21] Kadri, P.A. and Al-Mefty, O. (2004) Surgical Treatment of Dumbbell-Shaped Jugular Foramen Schwannomas. Neurosurgical Focus, 17, 56-62. https://doi.org/10.3171/foc.2004.17.2.9

[22] Chamoun, R., MacDonald, J., Shelton, C., and Couldwell, W.T. (2012) Surgical Approaches for Resection of Vestibular Schwannomas: Translabyrinthine, Retrosigmoid, and Middle Fossa Approaches. Neurosurgical Focus, 33, E9. https://doi.org/10.3171/2012.6.FOCUS12190 